\title{
Numerical methods for the dynamics of unbounded domains
}

\author{
EUCLIDES MESQUITA and RENATO PAVANELLO \\ Departamento de Mecânica Computacional, Universidade Estadual de Campinas, UNICAMP \\ C.P. 6122, 13083-970 Campinas, Brasil \\ E-mails: euclides@fem.unicamp.br/pava@fem.unicamp.br
}

\begin{abstract}
The present article discusses the relation between boundary conditions and the Sommerfeld radiation condition underlying the dynamics of unbounded domains. It is shown that the classical Dirichlet, Neumann and mixed boundary conditions do not fulfill the radiation condition. In the sequence, three strategies to incorporate the radiation condition in numerical methods are outlined. The inclusion of Infinite Elements in the realm of the Finite Element Method (FEM), the Dirichlet-to-Neumann (DtN) mapping and the Boundary Element Method (BEM) are described. Examples of solved dynamic problems in unbounded domains are given for the Helmholtz and the Navier operators. The advantages and limitations of the methodologies are discussed and pertinent literature is provided.
\end{abstract}

Mathematical subject classification: 78A40, 80M10, 80M15.

Key words: Sommerfeld radiation condition, Finite Element Method, Infinite Elements, Dirichlet-to-Neumann Mapping, Boundary Element Method.

\section{Introduction}

In many physics and engineering areas nature may be best represented or modeled by unbounded or semi-unbounded domains. The propagation of noise generated by a traffic route, the dynamic interaction of offshore facilities with the surrounding ocean, the description of waves propagating through the soil and generated at shock producing industrial facilities or subway lines, the electromagnetic modeling of antennas, represent only a few selected topics that requires considering the dynamics of infinite domains. 
The key issue in modeling the dynamic behavior of infinite domains is the idea that there is no energy source at very large distances from the region being analyzed. All energy sources are circumscribed within a limited domain. So physically, energy generated at the bounded domain flows, usually as waves, mechanical or electromagnetic, from the sources into the infinite domain and is not reflected. The unbounded domain is a perfect sink, where energy, in all its frequency content and forms, is consumed.

The withdrawal of energy from the bounded system into the unbounded domain is known as the Sommerfeld Radiation Condition (SRC). Let us resort to Arnold Sommerfeld words [1]: "The sources must be sources, and not energy sinks. Energy radiated from the sources must dissipate in the infinite; energy shall not flow from the infinite into the field singularities".

The present article discusses the relation between boundary conditions (BC) and the SRC. It will be shown that the classical Dirichlet, Neumann and mixed BCs do not satisfy the SRC. Further, three strategies to incorporate the SRC in numerical methods will be outlined. The inclusion of Infinite Elements in the realm of the Finite Element Method (FEM), the Dirichlet-to-Neumann (DtN) mapping and the Boundary Element Method (BEM) are described. Examples of solution for dynamic problems in unbouded domains are given for the Helmholtz and the Navier operators. The advantages and limitations of the methodologies are discussed and pertinent literature is furnished.

\section{Mathematical description of the Sommerfeld Radiation Condition}

Discussing the stationary acoustic wave propagation problem in unbounded domains governed by the Helmholtz operator $\nabla^{2} u+k^{2} u=0$, Sommerfeld [1] formulated the mathematical expression for the SRC:

$$
\lim _{r \rightarrow \infty} r^{(d-1) / 2}\left(\frac{\partial u}{\partial r}-i k u\right)=0
$$

In equation (1) the variable $r$ represents the distance from the origin and $d$ is the dimension of the problem $(d=1,2,3)$. It is tacitly understood that all processes have a harmonic time dependence according to the expression $\bar{u}(x, t)=u(x) \exp (-i \omega t)$. 
The obstacles for the implementation of the SRC in numerical solution methods is now addressed. Domain type methods, like the Finite Difference Method (FDM) and the Finite Element Method (FEM), require the discretization of the entire domain. The larger the domain, the larger the size of the resulting algebraic system. Clearly no unbounded domain can be discretized by these methods, since it would lead to infinitely large algebraic systems. So the FEM mesh must be truncated at some position. Now the question arises as to which $\mathrm{BC}$ should be placed at the outer boundary of the truncated mesh. This must be a perfectly energy transmitting BC, since all energy must be radiated into infinity and no part of it should be reflected back to the bounded mesh domain.

The $1-d$ Helmholtz operator, which governs linear acoustics and the dynamics of bars

$$
\frac{d^{2} u}{d x^{2}}+k u^{2}=0
$$

is used to exemplify the difficulty of imposing a perfectly radiating $\mathrm{BC}$ to a bounded mesh. Considering the time harmonic dependence $\exp (-i \omega t)$, the solution of the homogeneous equation (2) may be expressed as [21]:

$$
\bar{u}(x, t)=A_{1} \exp [i(k x-\omega t)]+A_{2} \exp [-i(k x+\omega t)]
$$

The first expression on the right-hand side of (3) represents an outgoing harmonic wave with circular frequency $\omega$ and amplitude $A_{1}$, travelling in the positive $x$-direction with wave number $k$. The second expression is an incoming harmonic wave of amplitude $A_{2}$. Imposing the SRC (1) on solution (3) leads to $A_{2}=0$. This is a rather obvious result because it eliminates the incoming part of the wave and consequently no energy is coming from the infinite or is reflected at the boundary towards the origin. Therefore a BC fulfilling the SRC (1) must enforce $A_{2}=0$.

The problem is that, in the usual FDM or FEM mesh, it is not possible to simulate the radiating $\mathrm{BC}(1)$. Consider the one-dimensional domain $(0 \leq$ $x \leq 1$ ) governed by equation (2). Boundary conditions must be prescribed to determine the constants $A_{1}$ and $A_{2}$, as well as the proper wave number $k_{n}$, $(n=1,2, \ldots)$. For all cases considered, unit Dirichlet BC will be applied at left-end of the domain, $u(x=0)=1$. Dirichlet and mixed BCs will be applied 
at the right-end of the domain, $x=1$. The ability of the right-end $\mathrm{BC}$ to model the SRC $\left(A_{2}=0\right)$ will be discussed. Figure 1 shows the addressed BCs.

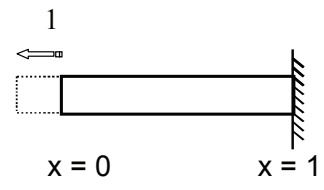

a) Case 1:

$u(x=0)=1$

$u(x=1)=0$

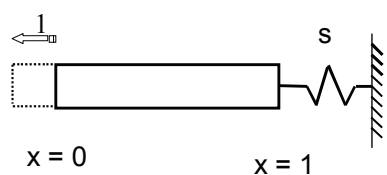

b) Case 2:

$u(x=0)=1$

$d u / d x=-\left.\mathrm{i} u\right|_{x=1}$

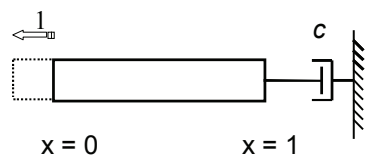

c) Case 3:

$u(x=0)=1$

$d u / d x=\left.\mathrm{i} \omega c u\right|_{x=1}$

Figure 1 - Boundary conditions for the 1-d Helmholtz operator.

Case 1. Homogeneous Dirichlet $\mathrm{BC}$ is applied at the right-end, $u(x=1)=0$. The solution is the superposition of two waves, with the complex amplitudes $A_{1}$ and $A_{2}=\left(1-A_{1}\right)$ determined at the proper wave numbers

$$
k_{n}=\frac{1}{2}\left[\frac{3 \pi}{4}+(n-1) \pi\right], \quad(n=1,2, \ldots) .
$$

As can be seen in figure 2, the solution for $k_{4}=\frac{15}{8} \pi$ is a standing wave (fig. 2a) composed of an outgoing wave with amplitude $A_{1}$ (fig. 2b) and superposed to an incoming wave $A_{2}=\left(1-A_{1}\right)$, (fig. 2c). This wave propagation pattern does not fulfill the SRC, since $A_{2} \neq 0$.

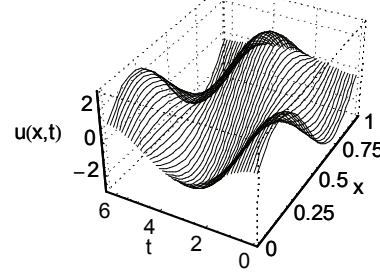

a) Resulting wave pattern: standing wave

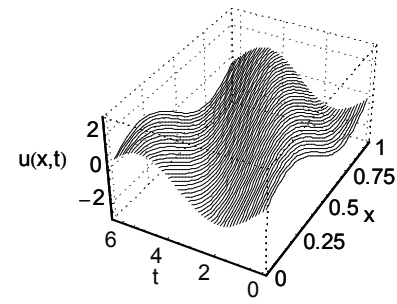

b) Outgoing wave $A_{1} \neq 0$

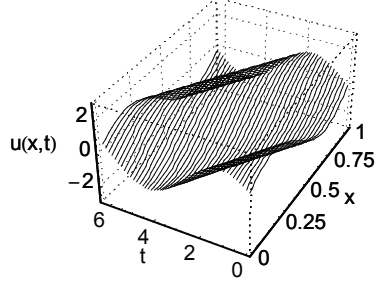

c) Reflected incoming wave $A_{2} \neq 0$

Figure 2 - Solution for homogeneous Dirichlet BC at the right-end, $u(x=1)=0$.

It can be shown that applying homogeneous Neumann BC to the right-end $d u(x=1) / d x=0$, will lead to a wave propagation pattern that has basically the same properties described in Case 1. 
Case 2. In the second case a spring with unit stiffness $(s=1)$ is applied at the right-end, leading to a mixed type $\mathrm{BC}, d u(x=1) / d x=-i u(x=1)$. Again the solution is the superposition of two waves, with the complex amplitudes $A_{1}$ and $A_{2}=\left(1-A_{1}\right)$ determined at the proper wave numbers. Figure 3 show the solution for $k_{4}=4,3831$ as a standing wave (fig. 3a) composed of an outgoing wave with amplitude $A_{1}$ (fig. $3 \mathrm{~b}$ ) and superposed to an incoming wave $A_{2}=\left(1-A_{1}\right)$, (fig. $3 \mathrm{c}$ ). Since $A_{2} \neq 0$, this wave propagation pattern also does not fulfill the SRC.

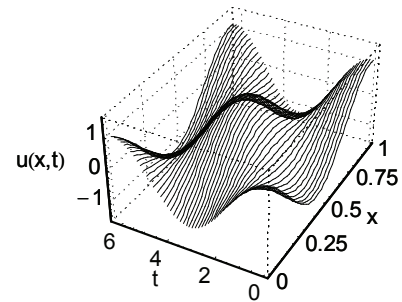

a) Resulting wave pattern: standing wave

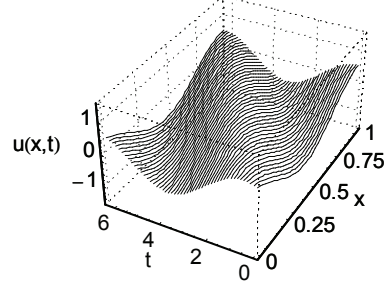

b) Outgoing wave $A_{l} \neq 0$

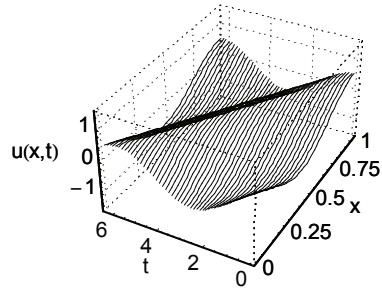

c) Reflected incoming wave $A_{2} \neq 0$

Figure 3 - Solution for mixed BC at the right-end, $d u(x=1) / d x=-i u(x=1)$.

Case 3. Mixed BC $d u(x=1) / d x=i \omega c u(x=1)$. This BC corresponds to a damper (dashpot) with a coefficient $c$ attached to the right-end of the domain. The solution of this problem has a particularity. For an arbitrary value of the damping coefficient $c$, the solution is a superposition of two waves with the complex amplitudes $A_{1}$ and $A_{2}=\left(1-A_{1}\right)$. But the damping value $c$ may be tuned according to the system characteristics to render a solution in which $A_{2}=0$. This solution is able to satisfy the SRC. The $c$ value which fulfills the $\mathrm{SRC}$ is called, in this context, the critical damping and is given by $c_{c}=k / \omega$, where $k$ is a system eigenvalue. The relation between the actual damping $c$ and the critical value $c_{c}$ is called damping factor, $f_{c}=c / c_{c}$.

Figure 4 depicts the wave propagation process for the wave number $k_{4}=$ 9.6566 and for a non-critical value of the damping, $f_{c}=0.60$. It can be readily recognized that the resulting wave propagation pattern (fig. $4 \mathrm{a}$ ) is the superposition of an outgoing wave (fig. 4b) and an incoming wave (fig. 4c). Clearly there is wave and energy reflection at the right-end boundary where the damper 
is attached and consequently $A_{2} \neq 0$. On the other hand the wave propagation for the critical value $f_{c}=1$ is shown in figure 5 .

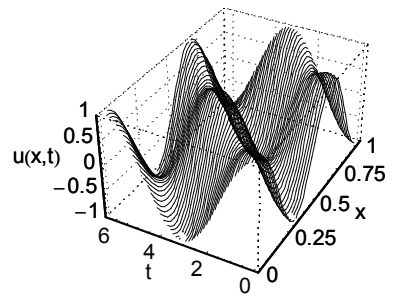

a) Resulting wave pattern: partially outgoing wave

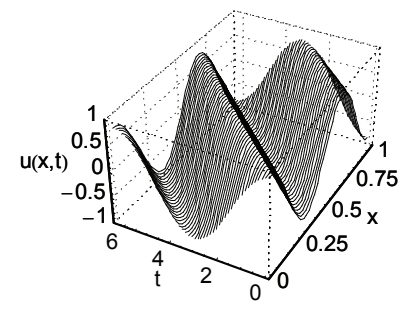

b) Outgoing wave $A_{l} \neq 0$

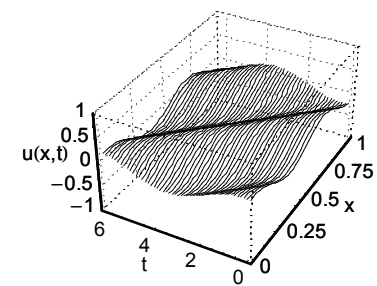

c) Reflected incoming wave $\mathrm{A}_{2} \neq 0$

Figure 4 - Solution for damper $c$ at $x=1$ : non-critical damping $f_{c}=0.60$.

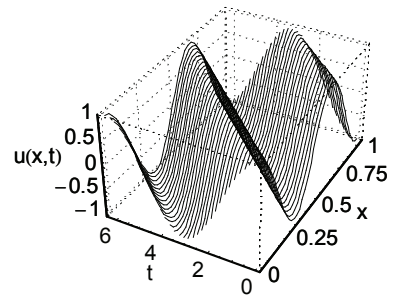

a) Resulting wave pattern: totally outgoing wave

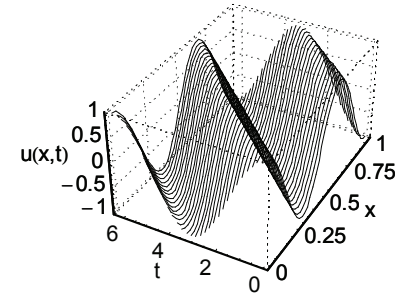

b) Outgoing wave $A_{1} \neq 0$

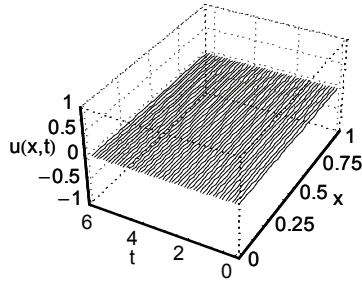

c) Reflected incoming wave $A_{2}=0$

Figure 5 - Solution for dashpot at $x=1$ : critical damping $f_{c}=1.0$.

But this is a very specific and unique situation. For one-dimensional, monochromatic propagation phenomena, there is a critical damping which may simulate the SRC. For two- and three-dimensional problems as well as for colored wave propagation processes, it is not possible to fulfill the SRC through one of the above described BCs.

For 1-d problems describing the axial and flexural waves in bars and beams, there is one element, called Spectral Element (SE), which may satisfy analytically the SRC [20]. The complex non-standing solution described in figure 4 represents a combination of a vibrating mode and a wave propagation phenomena. A detailed discussion of the relation between complex modes and wave propagation may be found in [22]. 
The key consequence to be drawn from this section is that there is no simple BC that can be applied to a bounded mesh in order simulated the SRC, which is inherently present in the dynamics of unbounded domains. The adequate numerical simulation of unbounded domain dynamics requires specific strategies which must be able to account for the SRC. In the sequence three such strategies will be described.

\section{Incorporating Infinite Elements in Finite Element Procedures}

Initial Remarks. The Finite Element Method (FEM) is the most widespread numerical method used to approximate the solution of problems in engineering and mathematical physics. The method is available in the form of commercial codes to a large part of the engineering community. So it would be desirable to develop a type of element which could model infinite (unbounded) domains and the underlying SRC. The developed elements have been called Infinite Elements (IEs) [32]. The inclusion of the IEs in the FEM code would provide a powerful enhancement to the method. They would fullfil the SRC and keep some important features of the original FE formulation.

Types of Infinite Elements. The pioneering articles of Bettess [30, 31, 32] introduced two classes of Infinite Elements, which have built the basis for their classification. According to Bettess [32] the Infinite Elements may be classified into:

- Decay Infinite Elements

- Mapping Infinite Elements

The basic idea in synthesizing an IE is to modify the standard FE shape functions $P_{i}(\varepsilon, \eta)$, expressed in terms of the normalized coordinates $\varepsilon, \eta$, by adding a decay or mapping function $F_{i}(\varepsilon, \eta)$. The resulting IE shape functions $N_{i}(\varepsilon, \eta)$ are $[32,40]$ :

$$
N_{i}(\varepsilon, \eta)=P_{i}(\varepsilon, \eta) F_{i}(\varepsilon, \eta)
$$


For monochromatic stationary wave propagating phenomena, the shape functions are modified by adding a decay parameter $\alpha$ and function with the wave number $k$ :

$$
N_{i}(\varepsilon, \eta)=P_{i}(\varepsilon, \eta) F_{i}(\varepsilon, \eta, \alpha) \exp (i k \varepsilon)
$$

In elastic solids, when compression $(p)$, shear $(s)$ and Rayleigh $(r)$ waves are present, the IE may include a shape functions for every wave type $(j=p, s, r)$

$$
N_{i}(\varepsilon, \eta)=\sum_{j} \lambda_{j} P_{i}(\varepsilon, \eta) F_{i}\left(\varepsilon, \eta, \alpha_{j}\right) \exp \left(i k_{j} \varepsilon\right)
$$

In this formulation the decay factors $\alpha_{j}$ and the coefficients $\lambda_{j}$ must be determined either empirically or using information from known analytical solutions. Several IEs have been proposed, which fall into this schematic representation $[10,37,48,46,49,47]$. Decay functions may present a polynomial or an exponential character and they may decay in one, two or three directions according to the problem requirements. Mapping functions may also be one-dimensional or multi-dimensional. The choice of the decaying or mapping function is largely determined by the numerical integration schemes available to synthesize infinite element matrices. Figure 6 represents a typical radially oriented $R(\varepsilon)$ Infinite Element. Figure 7 shows a two-dimensional exponential decay shape function, whereas figure 8 shows a typical two-dimensional mapping Infinite Element.

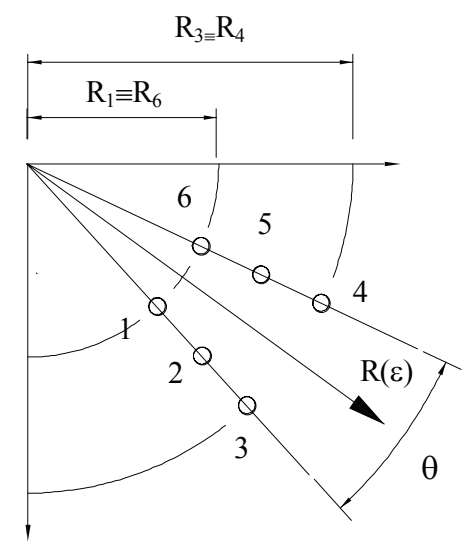

Figure 6 - A radially oriented $\mathrm{R}(\varepsilon)$ Infinite Element. 


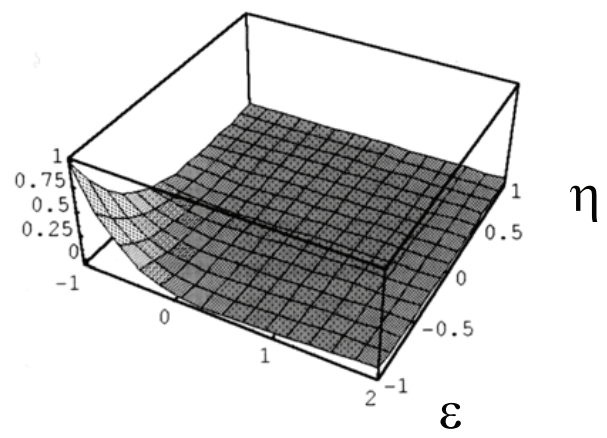

$\mathrm{F}(\varepsilon, \eta)$

Figure 7 - A typical two-dimensional decay Shape Function $\mathrm{F}(\varepsilon, \eta)$.

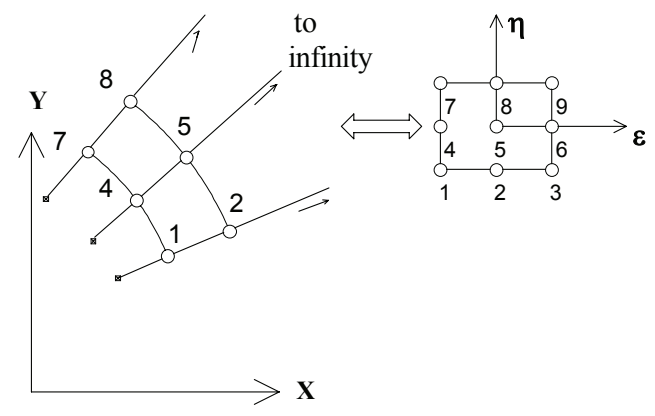

Figure 8 - A typical mapping infinite element.

Numerical examples. A Dynamic Soil-Structure Interaction (DSSI) problem will illustrate the versatility of the IEs incorporated to a standard FE code. Figure 9 shows a two-dimensional surface foundation interacting with a viscoelastic layer resting on a half-space. Figure 10 shows the Finite Element modeling of the problem. It can be seen that the foundation can be made rigid or flexible, by modifying its constitutive parameters, the layer can also be represented by changing the properties of the corresponding elements. Figure 10 also depicts the horizontal and vertical infinite elements at the soil boundary. The FE mesh has $16 \times 8$ Lagrangean quadratic elements with 9 nodes $(L / B=4.0)$ and 64 mapping Infinite Elements with 6 nodes (see fig. 8) [40]. The layer depth is $H / B=2.0$. The parameter $B$ is half of the foundation width. 


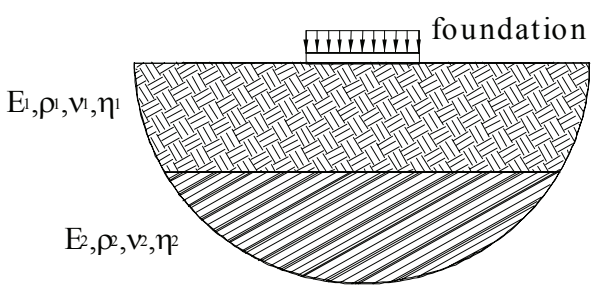

Figure 9 - A rigid foundation on a stratified soil model.

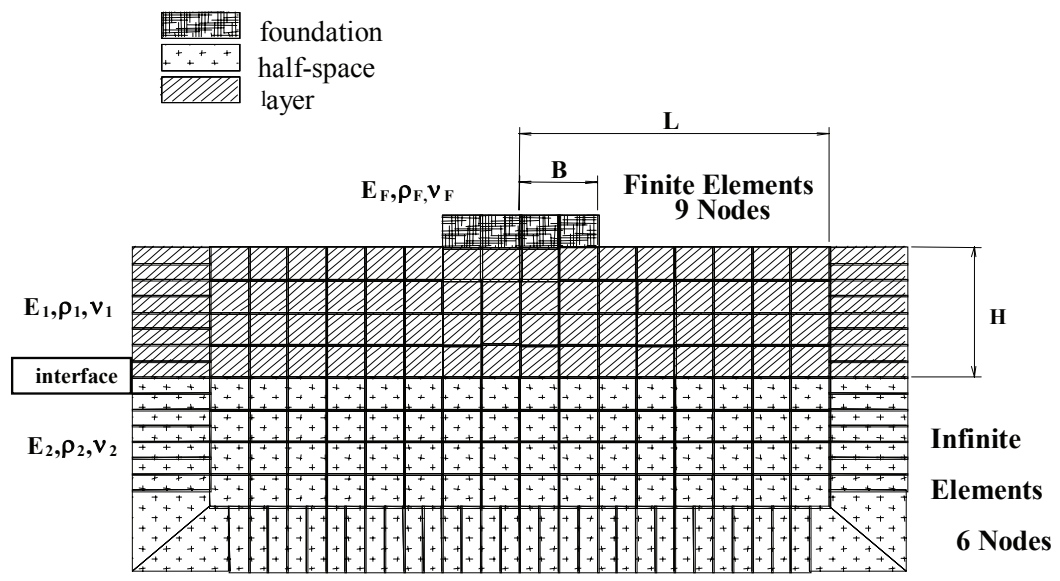

Figure 10 - Modeling of stratified soil with Finite and Infinite Elements.

In this problem the decay parameter for the compression and shear waves are adjusted to $\alpha_{p}=\alpha_{s}=0.2$. For the Rayleigh wave component the parameter is $\alpha_{r}=0.01$. The coefficients $\lambda_{j}$ were given values related to the energy carried by each type of wave in the homogeneous half-space [21], $\lambda_{p}=0.08, \lambda_{s}=0.25$, $\lambda_{r}=0.67$.

Figure 11 shows the Real part of the rigid foundation horizontal displacement due to a horizontal unit excitation (the compliance) $\operatorname{Re}\left(C_{v v}\right)$ as a function of the dimensionless frequency parameter $A_{0}=\omega B / c_{2}$. The shear wave velocity of the layer and half-space are, respectively, $c_{1}$ and $c_{2}$. The results for three distinct values of $c_{1} / c_{2}$ are compared to those obtained by Romanini [8] using a Green's function approach. The reported result shows that with the proper setting of parameters and coefficients, the Infinite Elements may be able to reproduce accurately the complex dynamics of a structure interacting with a stratified soil. 


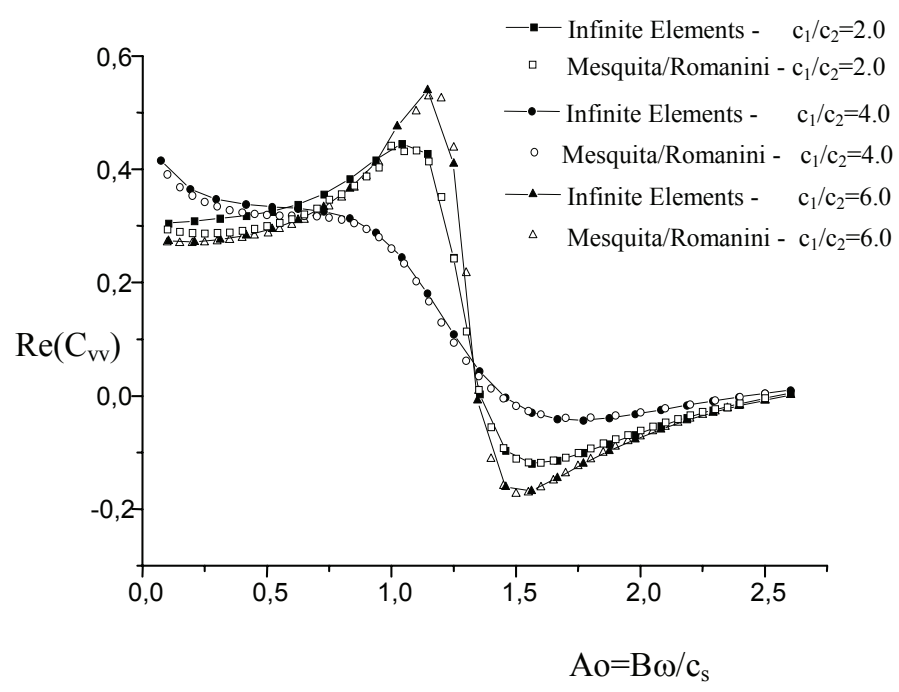

Figure 11 - Real part of rigid foundation vertical compliance $\operatorname{Re}\left[C_{v v}\left(A_{0}\right)\right]$, $H / B=2.0, v=0.25$.

Limitations and Drawbacks of the IE. In spite of the good results reported in this article the application of IEs presents a series of limitations:

1) The inclusion of the IE requires the development and implementation of special numerical integration techniques, which may be time consuming;

2) For monochromatic wave propagation phenomena, like linear acoustics, the IE requires the choice of a decay parameter for the shape function. The value of the decay parameter may be obtained from the asymptotic solution of the operator in unbounded domains. For colored wave propagation phenomena, where compression, shear, Rayleigh and Love waves may be present in the displacement field, a decay parameter $\alpha_{i}$ for each wave type is necessary. Another set of parameters $\lambda_{i}$, describing the distribution of energy among the several waves must be assigned to the IE shape functions. These parameters must be determined empirically or resort must be made to existing analytical solutions;

3) The application of IE, up to the present time, is restricted to the stationary analysis of the continua. There is no transient FE applications involving IE; 
4) The inclusion of IE in the FE codes requires involved variables to be extended to the complex case, increasing the amount of necessary storage.

\section{The Dirichlet-to-Neumann (DtN) Mapping and its incorporation in the FEM}

Initial remarks. The so called Dirichlet-to-Neumann (DtN) mapping is another strategy developed to incorporate de SRC into a FEM procedure. The method was first presented by Givoli and his co-workers [18, 5]. The basic idea is to divide the unbounded region in two domains by an artificial boundary. The first domain is a bounded one and is governed by a non-homogeneous operator. The second domain is unbounded and its corresponding operator is homogeneous. This second domain is subjected to Dirichlet boundary conditions at the artificial interface and the SRC (1) at infinity. An auxiliary analytical or numerical solution must exist for the problem posed on the second domain. The method, which, in the sequence, will be outlined based on the Helmholtz operator, has been intensively investigated $[13,14,15,16,17,25,6,7,28,29]$.

Formulation of the DtN mapping. The DtN procedure will be shown for a typical operator. Let us consider a body $B$ immersed in an infinite two-dimensional fluid $F$ as shown in Figure 12. The stationary behavior of the inviscid fluid $F$ is given in terms of the velocity potential $\psi$ governed by the Helmholtz operator. At the fluid-body interface, Dirichlet $\left(g\right.$ on $\left.\Gamma_{g}\right)$ and Neumann $\left(h\right.$ on $\Gamma_{h}$ ) boundary conditions are prescribed, with $\Gamma=\Gamma_{g} \cup \Gamma_{h}$. At infinity the SRC (1) is prescribed. Mathematically the problem can be stated as:

$$
\begin{aligned}
& \nabla^{2} \psi+k^{2} \psi+f=0 \quad \text { on } \quad F \\
& \psi=g \quad \text { on } \quad \Gamma_{g} \\
& \frac{\partial \psi}{\partial \eta}=i k h \quad \text { on } \quad \Gamma_{h} \\
& \lim _{r \rightarrow \infty}\left[r^{1 / 2}\left(\frac{\partial \psi}{\partial r}-i k \psi\right)\right]=0
\end{aligned}
$$

In equations (7) $r$ is the distance to the origin, $k$ is the wave number. Now, in the problem shown in Figure 12, the domain $F$ will be divided into two 


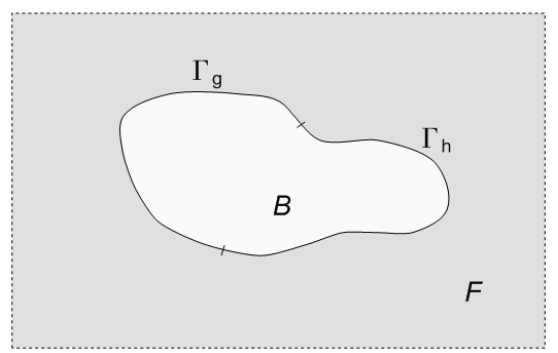

Figure 12 - Unbounded Fluid Domain $F$ surrounding a body $B$ at interface $\Gamma$.

distinct domains $F=D \cup \Omega$ connected by an artificially introduced boundary $\partial B_{R}$. The first domain $\Omega$ is bounded and is governed by the non-homogeneous operator, whereas the domain $D$ is unbounded and the corresponding operator is homogeneous, see figure 13. The problem on domain $D$ is a Dirichlet type problem and can be stated as:

$$
\begin{aligned}
\nabla^{2} \psi+k^{2} \psi & =0 & & \text { on } \quad D \\
\psi & =\psi(R, \theta) & & \text { on } \quad \partial B_{R} \\
\lim _{r \rightarrow \infty}\left[r^{1 / 2}\left(\frac{\partial \psi}{\partial r}-i k \psi\right)\right] & =0 & &
\end{aligned}
$$

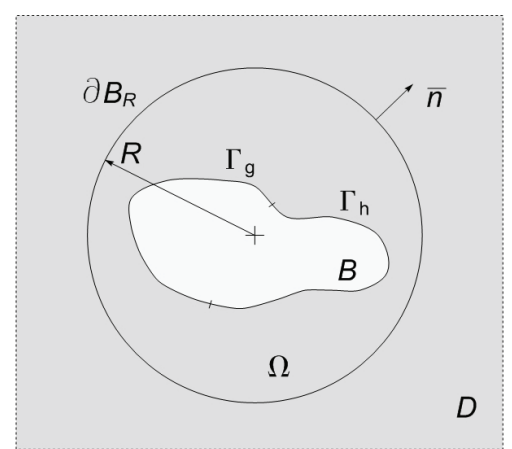

Figure 13 -Division of the problem in two domains by the artificial boundary $\partial B_{R}$.

The two-dimensional problem stated by (8) has an analytical solution as a series sum over $n$ harmonics [36, 28]:

$$
\psi(r, \theta)=\frac{1}{\pi} \sum_{n=0}^{\infty^{\prime}} \int_{0}^{2 \pi} \frac{H_{n}^{(1)}(k r)}{H_{n}^{(1)}(k R)} \cos n\left(\theta-\theta_{H}\right) \psi\left(R, \theta_{H}\right) d \theta_{H}
$$


In expression (9) $r$ is the radius of the point where the solution is being evaluated, $\theta$ is the angle related to the evaluating point, $\theta_{H}$ is a reference angle, $R$ is the radius of the artificial boundary, $H_{n}^{(1)}$ is the Hankel function of order $n$ and type 1 . The prime' indicates that the first term of the series $(n=0)$ should be multiplied by a factor $1 / 2$. Using expression (9) it is possible to establish a relation between the Dirichlet variable $\psi$ and its derivative with respect to the direction $r$, the Neumann variable $\partial \psi / \partial r$ :

$$
\frac{\partial \psi}{\partial r}=M \psi \quad \text { on } \quad \partial B_{R}
$$

This relation is called the Dirichlet to Neumann (DtN) mapping. Using equation (9) the DtN operator may be expressed as:

$$
M \psi=-\frac{k}{\pi} \sum_{n=0}^{\infty^{\prime}} \frac{H_{n}^{(1)^{\prime}}(k R)}{H_{n}^{(1)}(k R)} \int_{0}^{2 \pi} \cos n\left(\theta-\theta_{H}\right) \psi\left(R, \theta_{H}\right) d \theta_{H}
$$

Once the problem on the unbounded domain $D$ is solved, the counterpart on the bounded domain $\Omega$ may be formulated as a DtN problem. The DtN name stems from the fact the Neumann and Dirichlet conditions are related to each other on $\partial B_{R}$.

$$
\begin{aligned}
& \nabla^{2} \psi+k^{2} \psi+f=0 \quad \text { on } \quad \Omega \\
& \psi=g \quad \text { on } \quad \Gamma_{g} \\
& \frac{\partial \psi}{\partial n}=i k h \quad \text { on } \quad \Gamma_{h} \\
& \frac{\partial \psi}{\partial n}=M \psi \quad \text { on } \quad \partial B_{R}
\end{aligned}
$$

The problem described by equations (12) is restricted to a bounded domain and can be subjected to a classical FE formulation, as outlined in the sequence.

Incorporating the DtN mapping into the FE discretization. The Neumann BCs of the problem are included in the weak FE formulation through a boundary integral. Considering $w$ a weight function and the mapping in (10), the integral over the artificial boundary $\partial B_{R}$ may be written

$$
\int_{\partial B_{R}} w \frac{\partial \psi}{\partial n} d \Gamma=-\int_{\partial B_{R}} w M \psi d \Gamma
$$


Applying the Galerkin Method and the standard FE shape functions $N_{i}$ as the weighting function for the approximation of the velocity potential $\psi$, a discrete form of the integral (13) can be stated in matrix notation

$$
-\left[\int_{\partial B_{R}} N_{i} M N_{j} d \Gamma\right]\left\{\psi_{j}\right\}=[D]\left\{\psi_{\partial B_{R}}\right\}
$$

After matrix $[\mathrm{D}]$ is numerically determined, it can be incorporated in the discretized FE equations, representing the unbounded domain dynamics. Designating $[\mathrm{S}]$ and $[\mathrm{H}]$, respectively, the compressibility and volumetric matrices, the FE system may be written

$$
[S]\{\ddot{\psi}\}+([H]+[D])\{\psi\}=\{F\}
$$

Numerical examples. The described formulation has been implemented into a FE code to solve the problem of a harmonically vibrating cylinder of radius $r=a$. At the fluid-cylinder interface the Dirichlet boundary condition $\psi=\cos (4 \theta)$ was applied. The bounded $\Omega$ domain $(a<r<2 a)$ was discretized by three rows of 32 quadrilateral bi-linear elements, as shown in Figure 14. The artificial boundary $\partial B_{R}$ was place at $R=2 a$. The excitation frequency parameter was $k a=\pi$, and 6 finite elements were used for each wavelength.

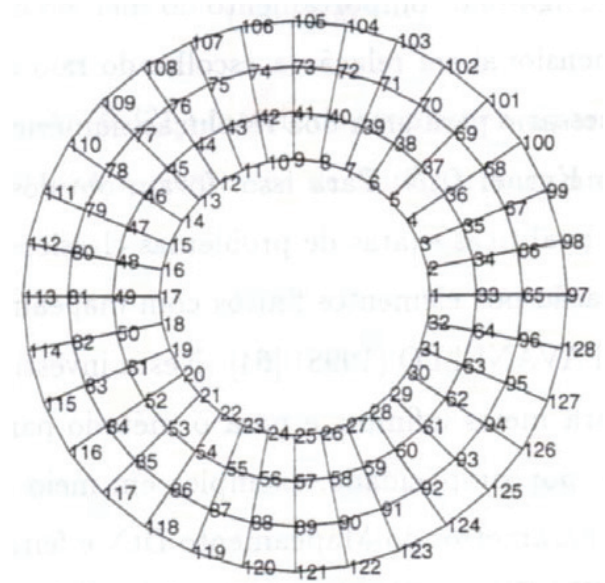

Figure 14 - FE mesh for an harmonically vibrating cylinder in unbounded fluid.

Figure 15 shows the imaginary part of the radiating harmonic field, compared to the exact solution given in [14]. For this problem only the first harmonic of the 
DtN operator $(n=0)$ needs to be implemented. The presented results indicate that the DtN mapping is an accurate tool to represent the stationary Sommerfeld radiation condition in unbounded domains.

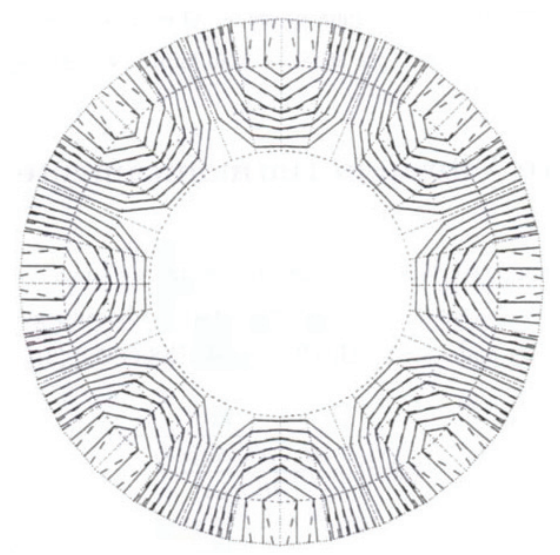

Figure 15 - Imaginary part of the radiating field from a harmonic vibrating cylinder: - Numerical, - - - Analytical.

Final remarks. Although accurate results have been obtained [28] and reported in the literature $[13,14]$ for the DtN mapping, some important aspects of procedure should be stressed:

1) The implementation of the DtN mapping requires a numerical or analytical solution of a Dirichlet type problem, in order to synthesize the DtN operator $M$;

2) The $M$ operator is a non-local operator and every node on the boundary $\partial B_{R}$ is connected to all other nodes. This leads to a block of fully populated matrix, which breaks the sparse and banded character of the original FE matrices;

3) The DtN operator introduces complex variables in the analysis increasing the need for storage and computing resources;

4) There are two parameters, the determination of which requires either numerical studies or empirical practice: a) the distance of the radius $R$ on which the artificial boundary $\partial B_{R}$ is placed and $\mathrm{b}$ ) the number of terms $\mathrm{n}$ 
which must be calculated for the series solution (11). The greater $R$, the larger the number of FE in the bounded mesh. High values of $n$ imply computing intensive matrices [D];

5) The wave number is explicitly present in the block matrix of the operator $M$ and it is not possible to formulate the problem as a standard FE eigenvalue problem.

\section{The Boundary Element Method}

Describing the BEM. The Boundary Element Method (BEM) is another numerical tool available to approximate the solution of boundary value problems. It is based on the discretization of Boundary Integral Equations (BIEs). One of its great advantages is that only the boundary of the domain under consideration needs to be discretized. The implication of this fact is that three-dimensional domains require a surface discretization, whereas two-dimensional domains lead to a line elements. Thus the algebraic systems stemming from the BEM are smaller than its FEM counterpart [24].

From the dynamics point of view the BEM is very inviting since it can naturally account for the SRC [19]. For completely unbounded domains or full-spaces, only the boundary containing the radiating source needs to be discretized. The BEM can also be applied very effectively to fracture mechanic problems. It can handle accurately high stress gradients and crack propagation can be modeled without domain remeshing [27].

The BEM has also some limitations or drawbacks when compared to the FEM:

1) The BEM leads to smaller algebraic systems than the FE discretization, but the systems are usually fully-populated and non-symmetrical;

2) The boundary integral involves singular kernels and methods to deal with these singularities must be implemented;

3) The BEM is a superposition method and thus well suited to linear problems, and special techniques required to deal with non-linear problems[24, 27]; 
4) The BEM is best applied to homogeneous continua. If non-homogeneity or inclusions are present, then resort to sub-domain techniques must be made [24];

5) To solve dynamic problems in unbounded domains by a boundary-only integral equation, the BEM requires an auxiliary state which fulfills the SRC.

In spite of this, there is growing evidence that the BEM is the most versatile numerical tool available to describe the stationary and transient behavior of homogeneous unbounded continua [3, 4]. The first dynamic formulation of the BEM was published by Cruse [41]. The transient BE formulation is due to Mansur and Brebbia [44, 45]. The method has been developed by many authors and is a mature technique to deal with acoustics and elastodynamic problems $[11,19,3,4,12]$.

Formulating and developing the BEM. The first step in the mathematical formulation of the BEM is to convert the differential equation (DE) governing the problem into a boundary integral equation (BIE). The Navier equations for frequency domain $(\omega)$ linear elastodynamics in terms of the Cartesian displacement component $u_{i}$ is:

$$
\mu u_{i, j j}+(\mu+\lambda) u_{j, j i}+\rho \omega^{2} u_{i}=0
$$

In equations (16) $\rho$ is the continuum density and $\mu, \lambda$, are Lame's constants. Using an auxiliary elastodynamic state, expressed in terms of displacement and traction field components $u_{i j}^{*}, t_{i j}^{*}$, and resorting to a reciprocal work theorem or Green's second vector identity, the domain equations (16) may be transformed into a boundary-only $(\Gamma)$ integral equation [19]

$$
u_{i}=\int_{\Gamma} t_{i j}^{*} u_{j} d \Gamma-\int_{\Gamma} u_{i j}^{*} t_{j} d \Gamma
$$

The key issue on this transformation is the auxiliary state $u_{i j}^{*}, t_{i j}^{*}$. It is only possible to transform the DE (16) into the BIE (17) if the auxiliary state satisfies the differential operator. In other words, to synthesize a BIE for a transient, 
viscoelastic and anisotropic problem a corresponding transient, anisotropic and viscoelastic auxiliary state is needed. Further, the BIE will only satisfy the Sommerfeld radiation condition if the auxiliary state also fulfills this requirement. These auxiliary states, also called "Fundamental Solutions" in the BEM context, do exist for classical operators. The Navier equations for stationary and transient linear elastodynamics [2] and the Helmholtz operator [36] possess a fundamental solution in closed analytical form.

A research area that has received attention in the last years is related to the synthesis of auxiliary states for more complex or involved operators, including anisotropy [33, 38, 39, 34, 26] poroelasticity [42], stratification [43] and transient viscoelastic behavior [23,9]. These auxiliary states may synthesized analytically or numerically. In the sequence typical advances in the synthesis of auxiliary states will be briefly addressed.

Auxiliary states for transient viscoelastic continua. This section will describe, exemplarily, the numerical synthesis of a transient viscoelastic half-space Green's function, which represents a necessary auxiliary state to analyze transient viscoelastic half-spaces by boundary integral procedures. The Green's function will, initially, be synthesized in the Fourier frequency domain. The transient solution is determined by an accurate numerical inverse Fourier transform. The viscoelastic effects in the continua may be completely characterized by the complex Lame constants

$$
u^{*}(\omega)=\mu_{1}^{*}(\omega)\left[1+i \eta_{\mu}(\omega)\right], \quad \lambda^{*}(\omega)=\lambda_{1}^{*}(\omega)\left[1+i \eta_{\lambda}(\omega)\right],
$$

where $\mu_{1}^{*}(\omega), \lambda_{1}^{*}(\omega)$ are the storage moduli and $\eta_{\mu}(\omega), \eta_{\lambda}(\omega)$ are the damping factors. The corresponding viscoelastic version of Navier equation (16) is:

$$
\mu^{*}(\omega) u_{i, j j}+\left[\mu^{*}(\omega)+\lambda^{*}(\omega)\right] u_{j, j i}+\rho \omega^{2} u_{i}=0
$$

The boundary conditions of the problem can be been in figure 16. The halfspace surface is excited at the origin by a Dirac's delta at $t=0, \delta(0)$. The frequency domains displacement solutions $u_{i}(\omega)$ are determined numerically by performing an improper integration over the wave number domain $k$ [34]

$$
u_{i}(\omega)=\int_{0}^{\infty} H_{i j}(\omega, k) t_{j}(k) \exp (i \omega k) d k
$$


Figure 17 shows a typical frequency domain displacement Green's function $u_{z}$, synthesized for large values of the frequency parameter Ao and for distinct values of the hysteretic damping coefficient $\eta$. The viscoelastic effects are clearly depicted in the figure. These frequency domain solutions will now be transformed into time domain solutions by means of the inverse Fourier transform. Details of the numerical inversion process may be found in [9].

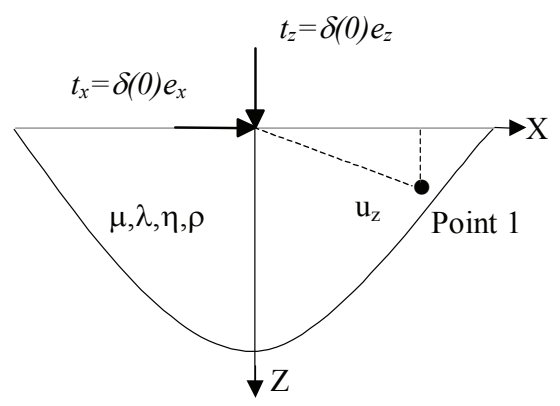

Figure 16 - Boundary Conditions for the half-space Green's function.

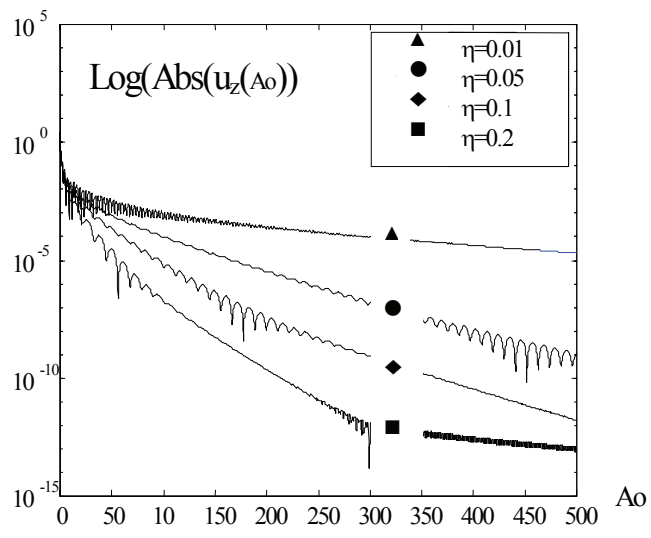

Figure 17 - Frequency domain solution for various damping parameters.

Figure 18 show the transient vertical displacement behavior $u_{z}(t)$ of an internal half-space point with coordinates $(x=1 \mathrm{~m}, z=1.73 \mathrm{~m})$ due to a vertical Dirac's impulse $\delta(x, z, t)$ applied at the origin $(x=0, z=0)$ at the instant $t=0$. This transient auxiliary state was obtained by inverse Fourier transformation of the solution presented in figure 17. 
The figure 18 also shows the influence of the viscoelastic constitutive parameter $\eta$. The viscoelastic transient auxiliary state was compared to the transient elastic solution presented by Richter [35]. The viscoelastic solutions tend to approach the elastic case as the damping factor $\eta$ is decreased. The arrival of the compression, shear and Rayleigh waves can be clearly seen in the transient result.

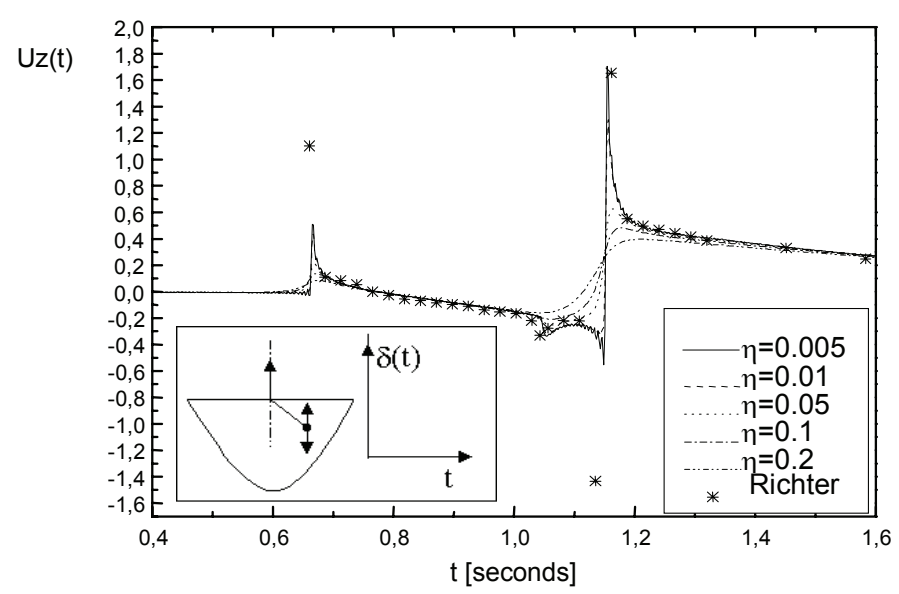

Figure 18 - Transient vertical displacement response $u_{z}(t, x=1 \mathrm{~m}, z=1.73 \mathrm{~m})$, due a vertical impulsive loading.

Figures 19 show a typical sequence of snapshots for the transient displacement field $u_{z}(t)$ at various time instants. An analysis of the obtained solutions show clearly that all wave propagation phenomena consists of outgoing and nonreflected waves. This auxiliary states clearly satisfy the Sommerfeld Radiation Condition.

This numerically synthesized transient viscoelastic solution may be incorporated into the BIE to render the transient viscoelastic solution of unbounded continua. It should be stressed that there is no general Fundamental Solution describing the transient behavior of viscoelastic continua. The reported results are original contributions to the implementation of the BEM to model transient behavior of viscoelastic continua. 


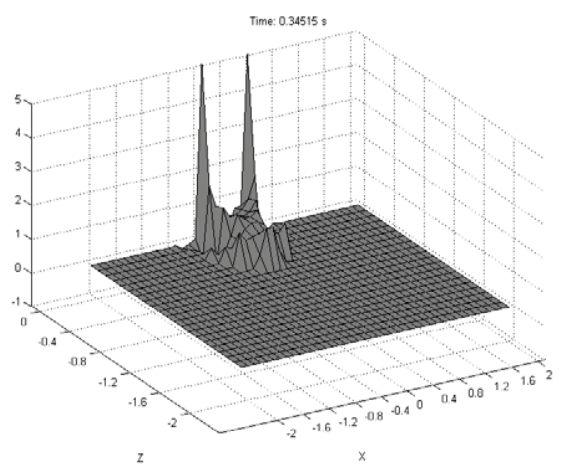

a) $\mathrm{t}=0.34515 \mathrm{~s}$

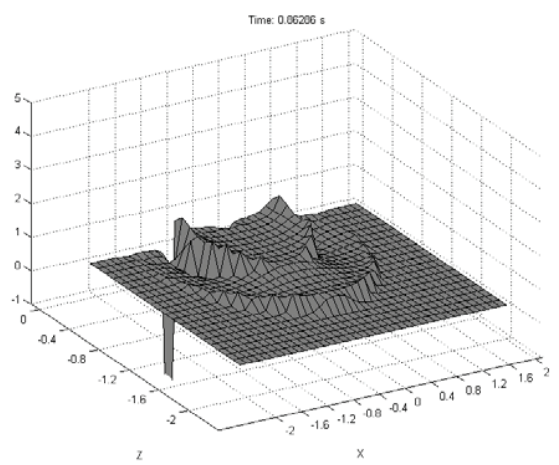

c) $\mathrm{t}=0.86296 \mathrm{~s}$

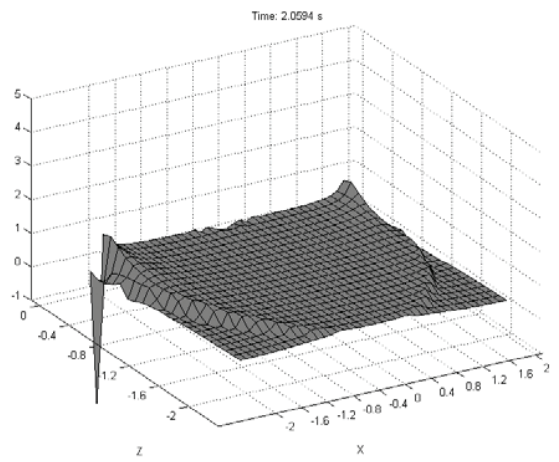

e) $\mathrm{t}=2.0594 \mathrm{~s}$

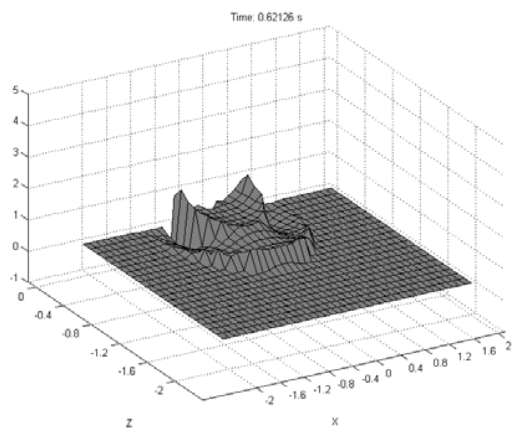

b) $\mathrm{t}=0.62125 \mathrm{~s}$

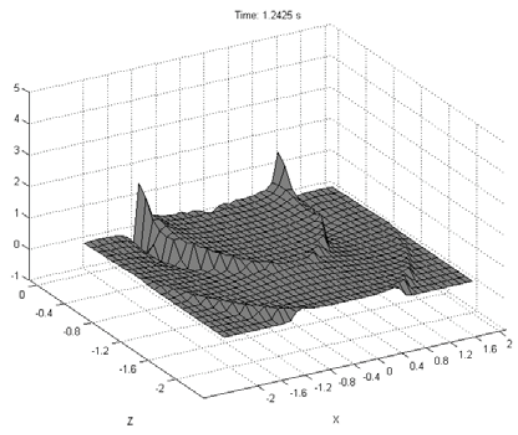

d) $\mathrm{t}=1.24250 \mathrm{~s}$ Trme $29990 \%$

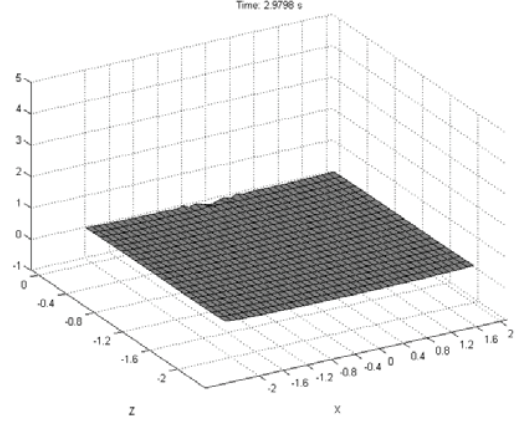

f) $t=2.9798 \mathrm{~s}$

Figure 19 - A snapshots of the transient viscoelastic auxiliary state $u_{z}(x, z, t)$. 


\section{Concluding Remarks}

The Sommerfeld Radiation Condition for unbounded domains and its relation to Boundary Conditions has been discussed. The main features, advantages and drawbacks of three numerical strategies, able to simulate the Sommerfeld Radiation Condition underlying the dynamics of unbounded domains, were described. Examples for the Helmholtz and Navier operator, satisfying the SRC were presented. A list of pertinent literature was furnished for each addressed methodology.

\section{REFERENCES}

[1] A. Sommerfeld, Partial Differential Equations, Academic Press, New York, (1949).

[2] A.C. Eringen and E.S Suhubi, Elastodynamics, vol.II, Academic Press, NY, (1979).

[3] D.E. Beskos, Boundary Element Methods in Dynamic Analysis, Applied Mechanics Reviews, 40 (1987), 1-23.

[4] D.E. Beskos, Boundary Element Methods in Dynamic Analysis: Part II (1986-1996), Applied Mechanics Reviews, 50 (1997), 149-197.

[5] D. Givoli, Numerical Methods for Problems in Infinite Domains, Elsevier, (1992).

[6] D. Givoli, I. Patlashenko and J.B. Keller, High-Order Boundary Conditions and Finite Elements for Infinite Domains, Computer Methods in Applied Mechanics and Engineering, 143 (1997), 13-39.

[7] D. Giljohann and M. Bittner, The Three-Dimensional DtN Finite Element Method for Radiation Problems of the Helmholtz Equation, Journal of Sound and Vibration, 212 (1998), 383-394.

[8] E. Romanini, Synthesis of Influence and Green's Functions for Dynamic Soil-Structure Interaction Problems by Boundary Integral Equations, PhD Thesis, Department of Computational Mechanics, State University at Campinas, (1995) (in Portuguese).

[9] E. Mesquita, M. Adolph, P.L.A. Barros and E. Romanini, Transient Green And Influence Functions For Plane Strain Visco-Elastic Half-Spaces, in: Proc. of the Iabem Symposium, The University of Texas at Austin, (2002), 1-12.

[10] F. Medina and R.L. Taylor, Infinite Elements for Elastodynamics, Earthquake Engineering and Structural Dynamics, 10 (1982), 699-709.

[11] G.D. Manolis and D.E. Beskos, Boundary Element Methods in Elastodynamics, Unwin Hyman Ltd., London, (1988).

[12] H.B. Coda and W.S. Venturini, Non-singular time-stepping BEM for transient elastodynamic analysis, Engineering Analysis with Boundary Elements, 15 (1995), 11-18. 
[13] I. Harari and T.J.R. Hughes, Finite Element Methods for the Helmholtz Equation in an Exterior Domain:Model Problems, Computer Methods in Applied Mechanics and Engineering, 87 (1991), 59-96.

[14] I. Harari and T.J.R. Hughes, Galerkin/Least-Squares Finite Element Methods for the Reduced Wave Equation with Non-Reflecting Boundary Conditions in Unbounded Domains, Computer Methods in Applied Mechanics and Engineering, 98 (1992), 411-454.

[15] I. Harari and T.J.R. Hughes, A Cost Comparison of Boundary Element and Finite Element Methods for Problems of Time-Harmonic Acoustics, Computer Methods in Applied Mechanics and Engineering, 97 (1992), 77-102.

[16] I. Harari and T.J.R. Hughes, Analysis of Continuos Formulations Underlying the Computation of Time-Harmonic Acoustics in Exterior Domains, Computer Methods in Applied Mechanics and Engineering, 97 (1992), 103-124.

[17] I. Harari and T.J.R. Hughes, Studies of Domain Based Formulations for Computing Exterior Problems of Acoustics, International Journal for Numerical Methods in Engineering, 37 (1994), 2935-2950.

[18] J.B. Keller and D. Givoli, A Finite Element Method for Large Domains, Computer Methods in Applied Mechanics and Engineering, 76 (1989), 41-66.

[19] J. Dominguez, Boundary Elements in Dynamics, Computational Mechanics Publications, Southampton, (1993).

[20] J.F. Doyle, Wave Propagation in Structures, $2^{\text {nd }}$ ed., Springer Verlag, New York, (1997).

[21] K.F. Graff, Wave Motion in Elastic Solids, Dover Publications, New York, (1975).

[22] K.M. Ahmida and J.R.F. Arruda, On the Relation Between Complex Modes and Wave Propagation Phenomena, Journal of Sound and Vibration, 254 (2002), 663-684.

[23] L. Gaul and M. Schanz, A comparative study of three boundary element approaches to calculate the transient response of viscoelastic solids with unbounded domains, Computational Mechanics, 179 (1999), 111-123.

[24] L.C. Wrobel, The Boundary Element Method, John Wiley \& Sons, (2002).

[25] M. Malhotra and P.M. Pinsky, A Matrix-Free Interpretation of The Non-Local Dirichlet-toNeumann Radiation Boundary Condition, International Journal for Numerical Methods in Engineering, 39 (1996), 3705-3713.

[26] M. Dravinski and Y. Niu, Three-dimensional time-harmonic Green's functions for a triclinic full-space using a symbolic computation system. International Journal for Numerical Methods in Engineering, 53 (2002), 455-472.

[27] M.H. Aliabadi, The Boundary Element Method, vol. 2, Applications in Solids and Structures, John Wiley \& Sons, (2002).

[28] P.A.G. Zavala, Vibro-acoustic Analysis Using the Finite Element Method and the Dirichlet to 
Neumann Mapping. MSc. Thesis, Department of Computational Mechanics, State University at Campinas, (1999) (in Portuguese).

[29] P.A.G. Zavala and R. Pavanello, Vibro-acoustic Modeling of Vehicle Interiors and Exteriors Using Finite Element Method, in: Proc. VII SAE Brazil Congress on Mobility, São Paulo, (1998), 1-7.

[30] P. Bettess, Infinite Elements, International Journal for Numerical Methods in Engineering, 13 (1977), 53-64.

[31] P. Bettess, More on Infinite Elements, International Journal for Numerical Methods in Engineering, 15 (1980), 1613-1626.

[32] P. Bettess, Infinite Elements, Penshaw Press, (1992).

[33] P.L.A. Barros and E. Mesquita, Elastodynamic Green's Functions for Orthotropic Plane Strain Continua with inclined Axis of Symmetry. International Journal for Solids and Structures, 36 (1999), 4767-4788.

[34] P.L.A. Barros and E. Mesquita, On the Dynamic Interaction and Cross-Interaction of 2D Rigid Structures with Orthotropic Elastic Media Possessing General Principal Axes Orientation. Meccanica, 36 (2001), 367-378.

[35] C. Richter, A Green's Function time-domain BEM of Elastodynamics, Computational Mechanics Publications, Southamptom, (1997).

[36] P.M. Morse and H. Feshbach, Methods of Theoretical Physics, McGraw-Hill, (1953).

[37] R.K.N.D. Rajapalske and P. Karasudhi, Efficient Elastodynamic Infinte Element, International Journal of Solids and Structures, 22 (1986), 643-657.

[38] R.K.N.D. Rajapakse and Y. Wang, Elastodynamic Green's Functions of Orthotropic Half Plane. ASCE, Journal of Engineering Mechanics, 117 (1991), 588-604.

[39] R.K.N.D. Rajapakse and Y. Wang, Green's Functions for Transversely Isotropic Elastic Half Space. ASCE, Journal of Engineering Mechanics, 119 (1993), 1724-1746.

[40] R.M. Barros, Infinite Elements to Model Stationary Viscoelastodynamics by the Finite Element Method, MSc Thesis, Department of Computational Mechanics, State University at Campinas, (1996) (in Portuguese).

[41] T.A. Cruse and F.S. Rizzo, A Direct Formulation and Numerical Solution of the General Transient Elastodynamic Problem -I, J. Mathematical Analysis and Applications, 22 (1968), 244-259.

[42] T. Senjuntichai and R.K.N.D. Rajapakse, Dynamic Green's Functions of Homogeneous Poroelastic Half-Plane. ASCE, Journal of Engineering Mechanics, 120 (1994), 2381-2404.

[43] T. Senjuntichai and R.K.N.D. Rajapakse, Exact Stiffness Method for Quasi-Statics of a Multi-Layered Poroelastic Medium, International Journal for Solids and Structures, 32 (1995), 1535-1553. 
[44] W.J. Mansur and C.A. Brebbia, Formulation of the Boundary Element Method for Transient Problems Governed by the Scalar Wave Equation, Applied Mathematical Modelling, 6 (1982), 307-311.

[45] W.J. Mansur and C.A. Brebbia, Numerical Implementation of the Boundary Element Method for transient problems goverved by the scalar wave equation, Applied Mathematical Modelling, 6 (1982), 299-306.

[46] Z. Chongbin and S. Valliapan, A Dynamic Infinite Element for-Three Dimensional InfiniteDomain Wave Problems, International Journal for Numerical Methods in Engineering, 36 (1996), 2567-2580.

[47] Z. Chuhan and O.A. Pekau and J. Feng, Application of FE-BE-IBE Coupling to Dynamic Interation Between Alluvial Soil and Rock Canyons, Earhquake Engineering and Structural Dynamics, 21 (1992), 367-385.

[48] Z. Chuhan and Z. Chongbin, Coupling Method of Finite and Infinte Elements for Strip Foundation Wave Problems, Earhquake Engineering and Structural Dynamics, 15 (1987), 839-851.

[49] Z. Chuhan and S. Chongmim, Infinite Boundary Elements for Dynamic Problems of the 3-D Half Space, International Journal for Numerical Methods in Engineering, 31 (1991), $447-462$. 